\title{
Author Index Vol. 7, 1997
}

Adragna,N.C. 251 Aiyar, J. 135 Ankorina-Stark, I. 321, 333

Beck,F.X. 119 Brenner, B. 107 Busch,G.L. 25

Cahalan, M.D. 133,219,229 Carnes, L. 251 Cermak, R. 321 Chandy,K.G. 135,243 Chang, M.C. 159 Chung, I. 159,312 Conley,E.C. 243 Costa Silva, V.L. 35

DeCoursey, T.E. 172 DeSmet,P. 19 Deetjen,P. 298 Dichgans,J. 282,304 Dolmetsch, R.E. 203 Dörge,A. 119 Droogmans, G. 19

Fanger,C.M. 203 Feidt,C. 264 Ferreira, A.T. 35 Fischer, R. 93 Fritzenwanger, M. 119 Fürst,J. 298

Garber,S.S. 229 Gibbons, N. 43 Grimmel,C. 304 Grissmer, S. 179 Gschwentner, M. 298

Gulbins,E. 107,148,282,304 Gutman, G.A. 135,243

Haller,T. 25 Hasegawa, T. 275 Häussinger, D. 93,289 Haxelmans, S. 321,333 Hayashi,Y. 304

Heinke, S. 19 Hirsch,J.R. 321,333 Hofer, S. 298 Hohage, H. 264 Hoth,M. 203

Ishiwari, K. 275

Jäger,H. 179

Kapasi, A. 43 Kawakatsu, H. 275 Kim,Y.K. 61 Kleta,R. 321 Ko,S.H. 61 Koch,F. 25 Komatsu, H. 275 Koppenhoefer, U. 107 Kubitz,R. 93,289

Lang,F. 25,107,148,282,303.

304 Lauf,P.K. 251

Lepple-Wienhues, A. 133,219 Lewis, R.S. 203 Lin,M. 81 Linderkamp, O. 107 Lohrmann, E. 333 López-Novoa, J.M. 69

Macías-Núñez, J.F. 69 Martínez-Salgado, C. 69 Moest,J. 25

Nagl,U.O. 298 Nelson, T.E. 81 Nilius,B. 19 Nishida,M. 275 Nishikawa, H. 275

Ortiz-Carranza, O. 251 Oshiro, M.E.M. 35

Park,K,M. 61 Paulmichl,M. 298

Pérez-Barriocanal, F. 69 Phipps,D.J. 312

Raskin, G. 19 Reddy,K. 43 Ritter, M. 25 Rodríguez-Barbero, A. 69 Rollbrocker, B. 304 Rühl,A. 93

Sawada,T. 275 Schlatter,E. 264,321,333 Schlichter, L.C. 159,312 Schmarda,A. 298

Schreiber,R. 289 Sharma,P. 43 Shelton,P.A. 243 Siemen, D. 25 Simon, M. 243 Singhal,M. 43

Singhal,P.C. 43 Stachon,A. 264 Strong, M. 135 Sudo,R.T. 81 Szabò,I. 148

Tamai,M. 275 Thurau,K. 119 Trepel,M. 304

Van Driessche, W. 19 Vasilets, L.A. 1 Verheugen, J.A.H. 188 Völkl,H. 25

Wagenknecht, B. 282, 304 Weinstock,C. 282 Weller,M. 282,304 Whalen, M.M. 53 Will,C. 282

Woo,J.S. 61

Zaladek Gil, F. 35 Zapata-Sudo, G. 81 Zweifach, A. 203

\section{KAHGER,}

Basel 
E-Mail karger@karger.ch Fax+ 41613061234 http://www. karger. ch 